\title{
Selective Removal of Penicillin G from Environmental Water Samples by Using Molecularly Imprinted Membranes
}

\author{
Meshude Akbulut Soylemez (D) \\ Hacettepe University, Department of Chemistry, Ankara, Turkey
}

\section{A BS T RACT}

Denicillin G imprinted membranes were prepared by utilizing UV induced in-situ polymerization. The characterization of membranes was conducted by using ATR-FTIR, XPS, SEM and AFM. The binding properties of imprinted membranes were evaluated against concentration and time. The binding capacity of the membranes was tested for real water samples. The size of the binding cavities of membranes was determined by using PALS. The specific selectivity of the membranes was investigated by using similar ß-lactams, penicillin $\mathrm{V}$ and amoxycillin. The specific binding of the membranes was determined as $3.27 \mu \mathrm{g} / \mathrm{g}$ for penicillin $\mathrm{G}$ while this value was obtained as 0.83 and $0.51 \mu \mathrm{g} / \mathrm{g}$ for pencillin $\mathrm{V}$ and amoxycillin, respectively. The binding capacity of the membrane was determined as $5.03 \mu \mathrm{g} \cdot \mathrm{g}^{-1}$ for ultra pure water while this value was obtained as $4.01 \mu \mathrm{g} \cdot \mathrm{g}^{-1}$ and $-3.50 \mu \mathrm{g} . \mathrm{g}^{-1}$ for tap water and natural water samples from different sources, respectively.

\section{Keywords:}

Membrane; Molecularly imprinted polymer; Penicillin G.

\section{INTRODUCTION}

ince its discovery, penicillin $\mathrm{G}$ has been useful for $\checkmark$ the treatment of infectious diseases in humans and animals. This compound is one of the most widely used drugs among antibiotics. Unfortunately, there is a continuous release of antibiotics due to the widespread and overuses of this family of the drug in some certain places such as hospitals and farms. Release of antibiotics to environmental waters is a crucial problem for human health. The residues of antibiotics were detected in industrial and municipal wastewater, and also surface and groundwater samples [1-4]. The residues of antibiotics in environmental waters may cause the development of antibiotic resistance [5]. The recognition and removal of the antibiotics from environmental water are necessary to protect public health by using easy, compatible, cheap and reliable materials.

There are several sensing and recognition systems for Penicillin G prepared by utilizing molecularly imprinting method [6-8]. Most of them are related by detecting of antibiotics from complex matrices such as food and food derivatives like milk, chicken, etc. $[8,9]$. The production methods includes synthesis of nanopolymers by emulsion polymerization [9], surface imprinting onto various support materials like magnetic nanoparticles [10] or non-woven fabrics [11]. Unfortunately, the number of researches on the removal of Penicillin G is not many. Molecularly imprinted polymers are functional materials prepared by polymerizing functional monomers and a crosslinking agent around a template molecule. Removing the template molecule from the network provides recognition sites that can accurately fit the template molecule concerning its affinity, size and shape. In this study, Penicillin G imprinted membranes were prepared by utilizing UV induced polymerization method for removal of the template molecule Penicillin G from real water samples. Photopolymerization is a good alternative to thermal polymerization by providing polymerization and crosslinking at room temperature. It is possible to say that the synthesis at room temperature is an important factor to get molecularly imprinted polymers with high specific selectivity depending on the stability of pre-polymerization complexes [10].

\section{EXPERIMENTAL}

\section{Materials}

Penicillin G, penicillin V, amoxicillin, methacrylic acid (MAA), ethylene glycol dimethacrylate (EGD MA), dimethylformamide (DMF), methanol, acetic acid and benzophenone were purchased from Sigma- 
Aldrich (Milwaukee, USA). All the reagents and solvents were used as received without any further purification step.

\section{Preparation of Penicillin G imprinted membranes}

Penicillin G (0.503 mmol) was dissolved in $2 \mathrm{~mL}$ DMF and then $2.01 \mathrm{mmol}$ MAA was added into the solution. The solution had been kept overnight at $4{ }^{\circ} \mathrm{C}$. EGDMA $(5.00 \mathrm{mmol})$ and $8 \mathrm{~mL}$ of DMF were added. The ratio of MAA to penicillin G was kept as 4:1 [11]. The formation of pre-polymerization complex between template and functional monomers and the synthesis of penicillin $G$ imprinted membranes were illustrated in Fig. S1. Finally, $20.0 \mathrm{mg}$ of benzophenone was added. The solution was purged with nitrogen and then placed between two identical silanized glass slides which separated by a rubber O-ring. The solution filled glass slides was placed into a zip-lock plastic bag under a nitrogen blanket. The polymerization was carried out by using a Philips type HB 171/A self-tanning UV lamp, adapted with four fluorescent tubes of Philips CLEO 15 W UVA. The irradiation time was kept as $90 \mathrm{~min}$. The distance from the glass slides to the UV lamp was held as $10 \mathrm{~cm}$. Subsequently, Penicillin G imprinted membranes (MIM) were carefully removed from the surface of the glass slides. The template molecule and unpolymerised monomers were washed out of the membranes with a mixture of acetic acid/methanol/water (1/5/1, by volume) until no Penicillin G was detected through a UV spectrometer. The non-imprinted membranes (NIM) was prepared using the same technique except for the addition of penicillin $G$.

\section{Characterization of membranes}

ATR-FTIR, XPS, SEM and AFM were used in the chemical and physical characterisation of membranes. The size of the recognition sites in the membranes was investigated by employing positron annihilation lifetime spectrometer (PALS) to analyze the effect of the presence of the template molecule.

The FTIR experiments were performed using Thermo Nicolet iS10 Thermo Nicolet iS10 model spectrometer in attenuated total reflection (ATR) mode. Spectra were recorded by accumulating 32 scans with a resolution of $4 \mathrm{~cm}^{-1}$.

Experiments with X-ray photoelectron spectroscopy (XPS) were carried out using a Thermo spectrometer with a monochromated $\mathrm{Al} \mathrm{K} \alpha \mathrm{X}$-ray source. Survey and core scans were reported with a pass energy of $30 \mathrm{eV}$ and $200 \mathrm{eV}$, respectively. All analyzes were performed at a take-off angle of $90^{\circ}$. Elemental composition of the surface of the membranes was examined with a $400 \mu \mathrm{m}$ X-ray spot size and binding energy ranged from 0-1000 eV.
SEM membrane images were taken using an FEI Quanta 200FEG instrument with an accelerating voltage of 2.00 $\mathrm{kV}$. Until screening, samples had been sputter-coated with gold.

Bruker Nanoscope 9 atomic force microscopy (AFM) was employed in tapping mode to make the morphological investigation of the membranes.

PALS experiments were performed using a standard system with detectors whose scintillators are plastic. The position of the detectors was face-to-face. The positron source was ${ }^{22} \mathrm{Na}$ with an activity of $11 \mu \mathrm{Ci}$, in the form of dried $\mathrm{NaCl}$ solution between two thin Kapton foils with glued together. The instrument time resolution was 258 ps (FWHM). The source was placed between two identical membranes in a typical 'sandwich' configuration. Each spectrum was saved in the air at room temperature every 3 $\mathrm{h}$ for a total count of $3 \times 10^{6}$. The sum of 5 spectra was used to produce $1.5 \times 10^{7}$ counts. The spectra obtained were examined using the program LT [12].

\section{Investigation of binding properties of membranes}

Batch binding experiments were performed to determine the binding capacity of imprinted and non-imprinted membranes. The membranes were incubated in $2 \mathrm{~mL}$ Penicillin $G$ solutions with a various concentration in the range of 0.5-50 ppm. The concentration of Penicillin G before and after incubation was analyzed by employing a UV-vis Varian Cary100 spectrophotometer at a maximum absorption wavelength of $216 \mathrm{~nm}$ [11]. The number of repetition of the analyses was three. The binding behaviour of membranes was analysed by using adsorption isotherms. The adsorbed amount of penicillin G (qe) was determined by using the following equation:

$$
q_{e}=\frac{C_{i}-C_{f}}{W} x V
$$

where $\mathrm{C}_{\mathrm{i}}$ and $\mathrm{C}_{\mathrm{f}}$ are the initial and equilibrium concentrations of template molecule, penicillin $\mathrm{G}(\mu \mathrm{mol} / \mathrm{L})$, respectively, $\mathrm{V}$ is the volume of solution (L), $\mathrm{W}$ is mass of the dry membrane (g).

The kinetic behaviour of adsorption was investigated by incubation for various periods in 3 ppm Penicillin G solution.

The reusability of the membranes was investigated by an adsorption-desorption cycle which was repeated 7 times. The concentration of Penicillin G was $3 \mathrm{ppm}$ and the incubation time was $20 \mathrm{~min}$. After each adsorption step, the membrane was washed as described before and then the incubation was repeated. 


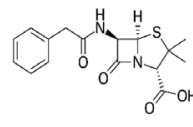

Penicillin $G$

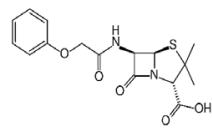

Penicillin $\mathrm{V}$

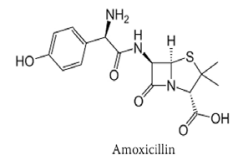

Figure 1. Chemical structures of antibiotics.

The specific selectivity of imprinted membranes was investigated by incubation in the presence of similar compounds, Penicillin V and amoxicillin (Fig. 1). The concentrations of antibiotics were kept as 3 ppm and the incubation time was $20 \mathrm{~min}$.

The binding performance of the membranes was investigated in real water samples collected from Pazar Creek (in Ankara) and Kızılırmak (in İskilip/Çorum region). And the binding performance of the membranes was also tested in tap water. Water samples were firstly filtered by using a 0.25 $\mu \mathrm{m}$ Nylon filter and stored at $+4{ }^{\circ} \mathrm{C}$ until analysis. Before experiments 3 ppm Penicillin G was spiked into the water samples. The incubation time was kept as $20 \mathrm{~min}$. All the experiments were repeated three times.

\section{RESULTS AND DISCUSSION}

\section{Chemical characterization of membranes}

As can be seen in Fig. 2 the structural characterization of imprinted and non-imprinted membranes was carried out by ATR-FTIR spectroscopy. The peak at $1721 \mathrm{~cm}^{-1}$ is attributed to stretching of $\mathrm{C}=\mathrm{O}$ groups of methacrylic acid and EGDMA. The stretching vibration of $\mathrm{sp}^{3}$ hybridized $\mathrm{C}-\mathrm{H}$ bonds can be seen at $2943 \mathrm{~cm}^{-1}$ while the bending vibration of the same group is at $1454 \mathrm{~cm}^{-1}$. The peak at $1140 \mathrm{~cm}^{-1}$ corresponds to the stretching of $\mathrm{C}$-O groups in dialkyl ethers [13]. All the peaks can confirm the chemical structure of poly(ethylene glycol dimethacrylateco-methacrylic acid). Unfortunately, it is not possible to see any evidence of the presence of the template molecule due to its trace amount in the polymeric network by using FTIR. However, the presence of the template molecule can be proven by using more sensitive instrumental analysis methods such as XPS. XPS is a useful tool which provides detailed information about the chemical composition of the surfaces after modification for the approximately $10 \mathrm{~nm}$ depth from the surface.

Therefore, the surface elemental composition of membranes was investigated by employing XPS. The survey scans of MIM and NIM can be seen in Fig 3. The chemical compositions of the imprinted and non-imprinted membranes show similarities for $\mathrm{C} 1 \mathrm{~s}(285 \mathrm{eV})$ and $\mathrm{O} 1 \mathrm{~s}$ $(532 \mathrm{eV})$ peaks with an exception of the N1s peak at 400 $\mathrm{eV}$ for MIM [14]. The appearance of N1s peak proves the presence of the template in the crosslinked polymer.

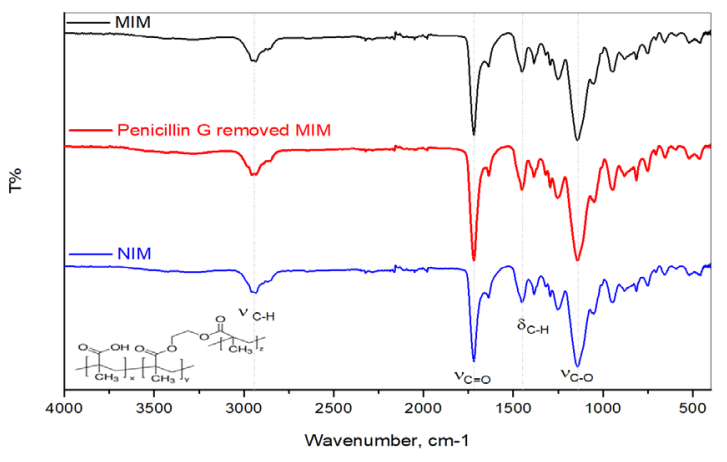

Figure 2. FTIR spectra of the imprinted membrane (MIM), template removed imprinted membrane and non-imprinted membrane (NIM).

The C1s core level spectra of MIM, pencillin G removed MIM and NIM can be seen in Fig 4. The common and the major component for all is the $\mathrm{C}-\mathrm{C}$ and $\mathrm{C}-\mathrm{H}$ peak which is detected at 284. eV. The component at $286.0 \mathrm{eV}$ is attributed to the $\mathrm{C}-\mathrm{O}$ [15]. The highest binding energy value $(\sim 288.3 \mathrm{eV})$ can be ascribed to $\mathrm{C}=\mathrm{O}$ groups of copolymeric matrix [14]. There is one more component in MIM spectrum at $287.4 \mathrm{eV}$ which corresponds to $\mathrm{C}-\mathrm{N}$ type carbon atoms in amide (399.9 eV) and lactam groups $(400.2 \mathrm{eV})$ of pencillin G (Fig. S2) [15]. This is a direct evidence of the presence of the template molecule in the imprinted membrane. It can be said that the procedure for the removal of template molecule is sufficient as can be noticed with the disappearance of $\mathrm{C}-\mathrm{N}$ in the spectrum of penicillin G removed MIM. The binding ability of the MIM was also investigated by XPS analysis. The C-N peak at 287.4 eV appears again for the penicillin $\mathrm{G}$ removed MIM after penicillin $\mathrm{G}$ incubation in 3 ppm for $20 \mathrm{~min}$.

\section{Physical and morphological characterization of membranes}

The presence of the template molecule in the imprinted polymers can cause various morphological differences [16]. The difference in the morphological structures of the surfaces of NIM and MIM can be seen from SEM

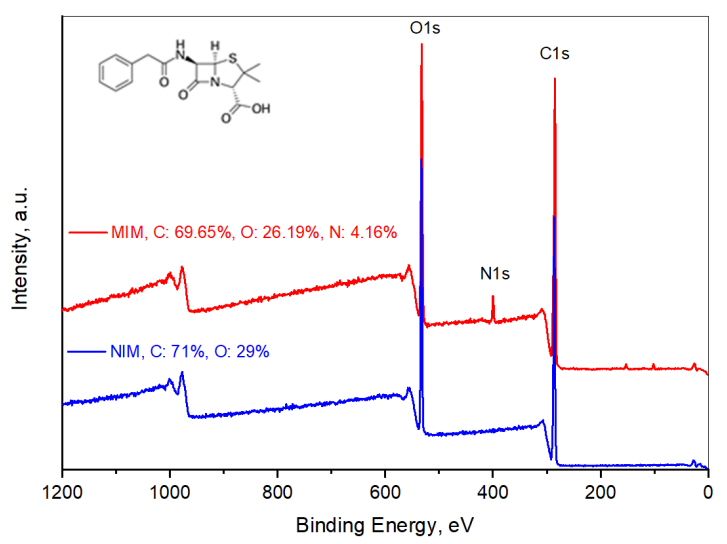

Figure 3. Survey scans of penicillin G imprinted (MIM) and nonimprinted membranes (NIM) and the chemical structure of penicillin G. 

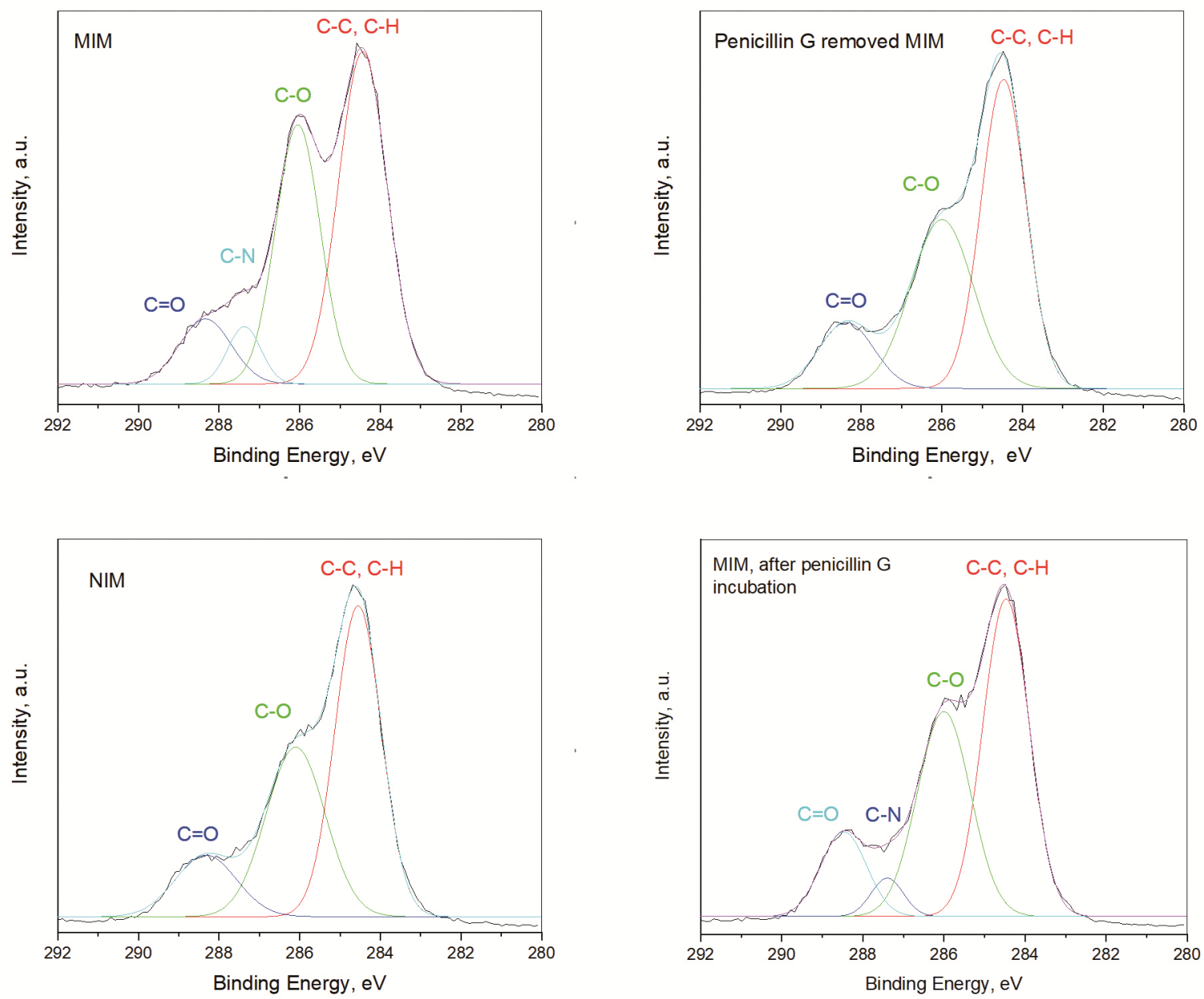

Figure 4. C1s spectra of penicillin G imprinted membranes (MIM), penicillin G free imprinted membranes by washing with a solution of acetic acid:methanol:water, non-imprinted membranes (NIM) and penicillin G free imprinted membranes after incubation in 3 ppm penicillin G solution for $20 \mathrm{~min}$.

images (Fig. 5). The interaction between the monomer and the template can cause more porosity and roughness on the surface of the membranes [17].

The roughness of the surfaces of the membranes was also investigated by employing AFM (Fig 6). The value of Rq is determined as 234 and 98 for MIM and NIM, respectively. The roughness of the surface of MIM provides higher binding capacity for penicillin $\mathrm{G}$ due to the increasing in surface area and permeability of the membranes that provides to

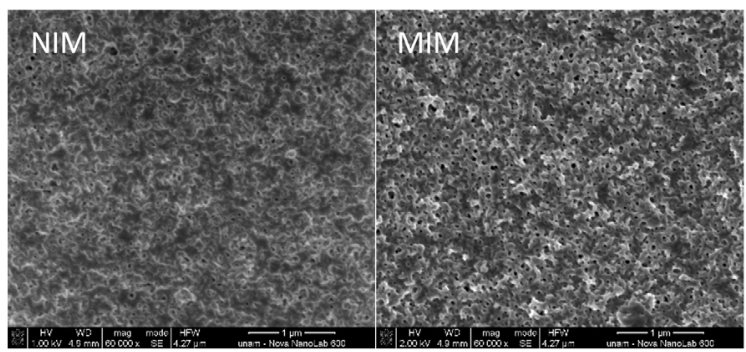

Figure 5. SEM images of NIM and MIM. enhance in the possibility of the interaction between MIM and penicillin $\mathrm{G}$ [18].

The size of the free volume holes in the membranes was investigated by employing PALS. It is an absolute and highly sensitive method which provides detailed and certain information on the size and size distribution of free volume holes in polymeric structures $[19,20]$ and defects in metals [21]. The selectivity of the molecularly imprinted polymers can be controlled mainly by two factors: 1) chemical affinity of the recognition sites and 2) binding cavity size and size
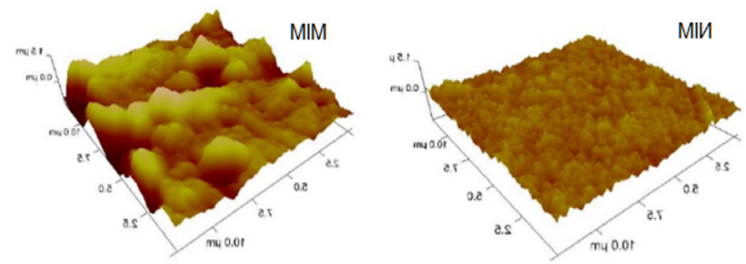

Figure 6. AFM images of non-imprinted (NIM) and imprinted membranes (MIM). 
distribution [13]. The second factor gains more importance when the binding of compounds with a similar molecular size is considered. Therefore, it is important to have information about the size of the binding cavities of imprinted polymers. In previous work from our laboratory, the average diameter of the free volume holes of imprinted polymers of penicillin $\mathrm{G}$ prepared by grafting on the non-woven fabric was determined as $0.570 \mathrm{~nm}$ while this value was obtained as $0.469 \mathrm{~nm}$ for non-imprinted polymer grafts [13]. In this study, the diameter of the free volume holes in MIM was obtained as $0.558 \pm 0.003 \mathrm{~nm}$ that matches to previously obtained data. The pore size of the NIM $(0.434 \pm 0.002 \mathrm{~nm})$ was also similar to the previous data for the control polymer. The obtained data also correlates to the molecular diameter of the penicillin $\mathrm{G}$ which was reported as $0.500 \mathrm{~nm}$ by Wu and coworkers [22].

\section{Investigation of the binding properties of membranes}

\section{Adsorption isotherms}

To examine the binding characteristics of the membranes binding isotherms were constructed. Adsorption isotherms are created by using the equilibrium concentration of an analyte onto an adsorbent $\left(\mathrm{q}_{\mathrm{e}}\right)$ and the concentration of the analyte in the solution $\left(\mathrm{c}_{\mathrm{e}}\right)$ with which it is in contact. This relationship is explained by using several equations. The most common models used to investigate the binding behaviours of the molecularly imprinted polymers are Langmuir, Bi-Langmuir and Freundlich isotherms (Table S1) [23]. The binding isotherms Langmuir and Freundlich were used to determine the binding
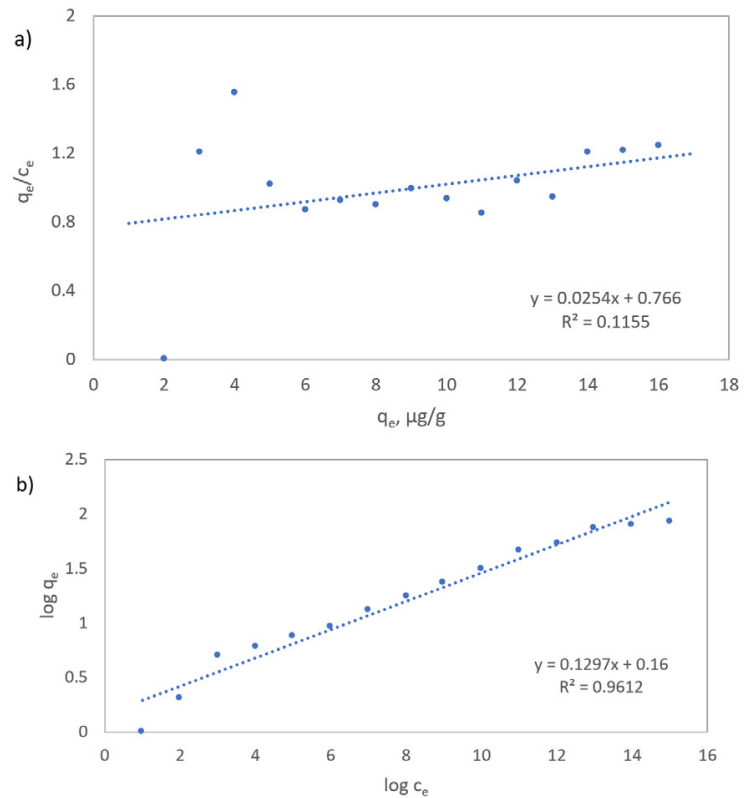

Figure 7. a) Langmuir and b) Freundlich isotherms for molecularly imprinted membranes (MIM). efficiency of the membranes (Fig. 7). As it is apparent the binding behaviour of MIM fits Freundlich model with an $\mathrm{R}^{2}$ value of 0.96 . Heterogenous binding models are more suitable to evaluate the binding behaviours of imprinted polymers due to their heterogeneous nature of the recognition sites [23]. The heterogeneity index, $\mathrm{n}$ which varies in the range of $0-1$ was determined as 0.13 . The value of $n$ shows an increase with an increase in homogeneity of the system and it equals to the 1 for an ideal homogeneous system [24]. As can be noticed the MIM prepared by conventional free radical polymerization method has a heterogeneous nature due to the polymerization method [25].

\section{Kinetics of adsorption of penicillin G imprinted membranes}

The kinetic evaluation of the adsorption is important as the type of the adsorption gives information about the nature of the binding. The adsorption rate-limiting step can be managed by diffusing the template to the adsorbent surface, or by the chemical interaction between the template and the adsorbent. Generally, the binding in molecularly imprinted polymers is controlled by chemical affinity between template and host. The kinetics of adsorption was evaluated by using Lagergren's first-order rate equation and pseudo-second-order rate equation (Table S2). The kinetic behaviour of the prepared membranes matches the pseudo-second-order kinetic model with an $R^{2}$ value of 0.99 (Fig. 8), which suggests that the rate-determining step of penicillin $\mathrm{G}$ adsorption is regulated by the chemical affinity of penicillin G to MIM [16, 26].
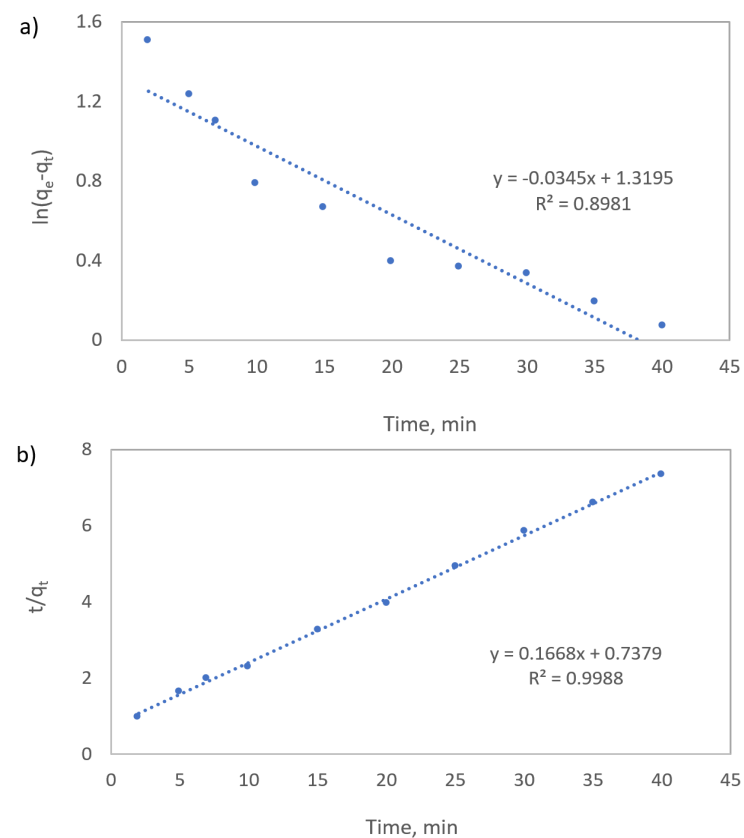

Figure 8. Curves of (a) first-order and (b) pseudo-second-order kinetic models (incubation concentration of penicillin $\mathrm{G}$ was $3 \mathrm{ppm}, \mathrm{n}=3$ ). 


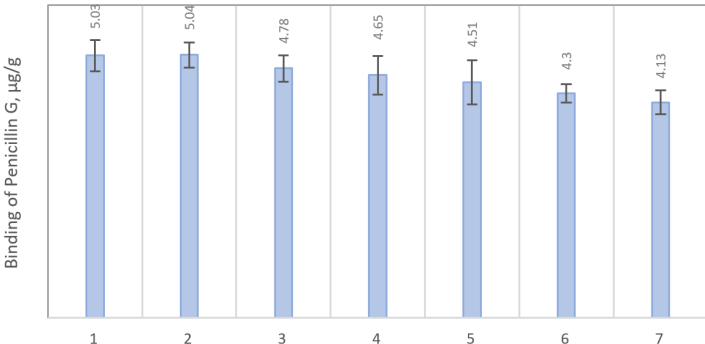

Figure 9. Binding of penicillin $\mathrm{G}$ which was determined by using the same imprinted membrane after each adsorption for seven cycles (incubation concentration of penicillin $\mathrm{G}=3 \mathrm{ppm}$, incubation time $=20 \mathrm{~min}$, $\mathrm{n}=3)$.

\section{Reusability of the membranes}

The reusability of the membranes was investigated by repeating the adsorption-desorption cycle 7 times (Fig 9). After each binding step, the removal of the template molecule was achieved by solvent extraction which was described previously. The performances of the imprinted membranes did not show a significant decrease upon repeated use. The crosslinked imprinted membranes can be used for many times without a significant binding capability loss.

\section{The binding capability of imprinted and non-imprinted membranes}

As is evident from Fig. 10 the adsorption capacity of MIM is greater than the binding capacity of NIM. The morphological differences and the data about the size of the recognition sites support the higher binding capability of the MIM. The roughness of the surfaces of the MIM enhances the chemical interaction between penicillin $G$ and the imprinted membranes. It can be assumed that the rise in surface area due to the roughness makes the availability of the binding sites easier [18]. Non-imprinted membranes (NIM) were produced without the addition of the template molecule using the same procedure. The absence of a template molecule produces smoother surface due to the lack of any specific chemical interaction [16]

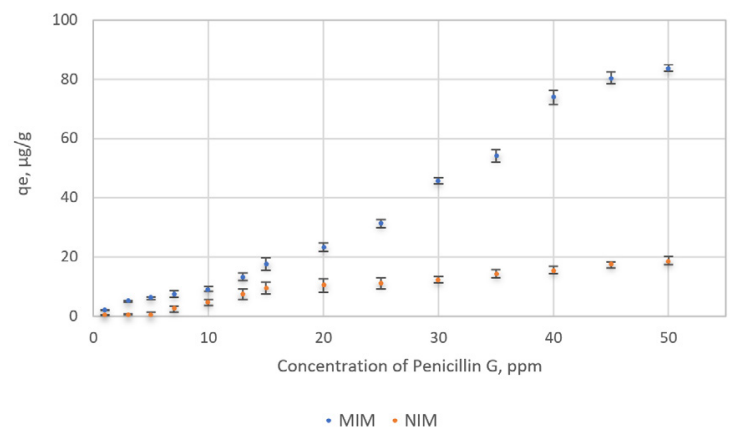

Figure 10. The adsorption capacity of imprinted (MIM) and nonimprinted membranes (NIM) ( $\mathrm{n}=3$, incubation time $20 \mathrm{~min}$ ).

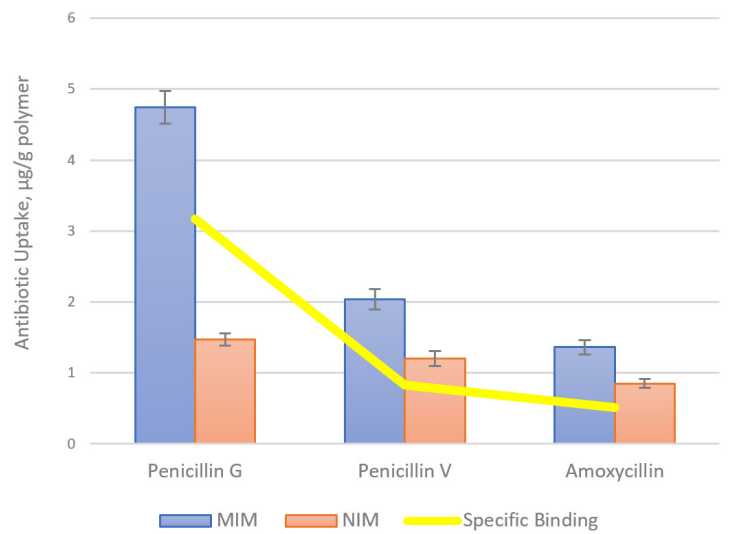

Figure 11. Selectivity of penicillin G imprinted (MIM) and nonimprinted membranes (NIM) against penicillin V and amoxicillin (concentration of each antibiotic $=3 \mathrm{ppm}$, incubation time $=20 \mathrm{~min}, \mathrm{n}=3$ ).

It still has a porous structure because of the presence of a porogen solvent.

\section{Specific selectivity of membranes}

Specific selectivity of the membranes was investigated by using structurally similar $\beta$-lactam antibiotics such as amoxicillin and penicillin V (Fig. 11). The concentration of the antibiotics was kept as 3 ppm for 20 min incubation. The specific binding (SB) value is a criterion to investigate the specific selectivity of the imprinted polymers against the target molecule. SB is determined by substracting of the binding onto non-imprinted polymers due to the non-specific interactions from the binding of imprinted polymers. Thus, the obtained binding data is the result of the specific interaction between the template and adsorbent [27]. SB of the MIM was determined as $3.27 \mu \mathrm{g} / \mathrm{g}$ polymer for penicillin $\mathrm{G}$ while this value obtained as 0.83 and $0.51 \mu \mathrm{g} / \mathrm{g}$ polymer for penicillin $\mathrm{V}$ and amoxicillin, respectively. Because of the complete complement of the binding sites in terms of shape, size and unique interaction between the template molecule penicillin $\mathrm{G}$ and the imprinted membrane, the highest SB value was obtained for the template molecule [11].

The selectivity (k) and relative selectivity coefficients $\left(k^{\prime}\right)$ were determined by using equations summarized in Table S3. The selectivity coefficient (k) is used to investigate the degree of selectivity of molecularly imprinted and non-imprinted polymers towards the target molecule. The selectivity of the MIM for penicillin G is 2.33 and 3.48 times higher than the selectivity towards penicillin $\mathrm{V}$ and amoxycillin, respectively (Table S4). The relative selectivity coefficients were determined as 1.38 and 1.46 for penicillin $V$ and amoxycillin, respectively. The data prove that the specific selectivity of MIM is higher than the NIM as expected due to the formation of binding sites by templating of penicillin G. 


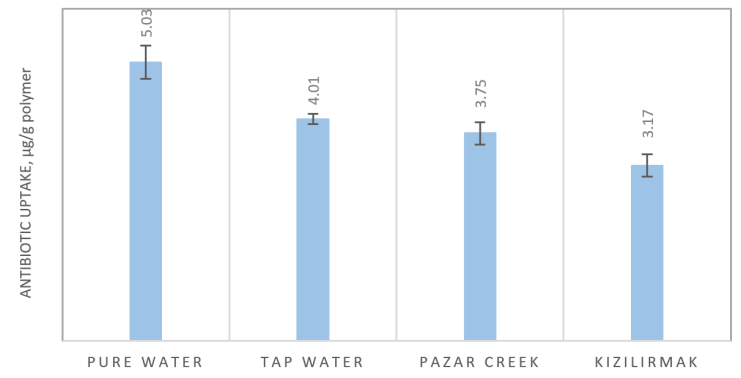

Figure 12. Binding performance of the imprinted membranes in real water samples.

\section{Investigation of the binding capacity in real samples}

To analyze the binding capabilities of the imprinted membranes in real samples tap water and natural surface water samples from different regions were used. As it is apparent in Fig. 12 the binding capacities of the MIM decreases due to the complexity of the real samples [16]. The binding capacity of the MIM was determined as 4.01 $\mu \mathrm{g} . \mathrm{g}^{-1}$ for tap water and $\sim 3.50 \mu \mathrm{g} \cdot \mathrm{g}^{-1}$ for natural surface waters which contains 3 ppm spiked penicillin G, respectively. It is possible to say that the imprinted membranes can be useful to remove penicillin $\mathrm{G}$ from real water samples.

\section{CONCLUSION}

Penicillin G imprinted membranes were produced by UV induced in-situ polymerization of methacrylic acid in the presence of a crosslinking agent. The detailed structural and physical characterization of the membranes was carried out using various methods of spectroscopy and microscopy. XPS was used to prove the existence of the template inside the imprinted membranes. The change in morphology of the membranes was investigated by SEM and AFM. The interaction of the template molecule and the network results in the roughness of the imprinted membrane surface. The size of the free volume holes of the imprinted membranes was investigated by PALS. The kinetic behaviour of the imprinted membranes supports the presence of the chemical affinity between penicillin $\mathrm{G}$ and the membranes. The binding capability of the membranes was investigated for real water samples. The binding capacity was determined as approximately 3.50 $\mu \mathrm{g} . \mathrm{g}^{-1}$. As a result, the prepared membranes can be useful to remove penicillin $\mathrm{G}$ from natural surface water samples. The use of the molecularly imprinted membranes as a filtration material for the selective removal of penicillin $G$ from environmental water sources can be considered as the main novelty of this work.

\section{ACKNOWLEDGEMENT}

I would like to thank Prof. Dr. Olgun Güven for his continuing support and encouragement throughout my studies.

\section{References}

1. Junker T, Alexy R, Knacker T, Kümmerer K. Biodegradability of 14C-Labeled antibiotics in a modified laboratory scale sewage treatment plant at environmentally relevant concentrations. Environmental Science \& Technology 40 (2006) 318-324.

2. Giger W, Alder AC, Golet E, Kohler HPE, McArdell CS, Molnar E, Siegrist H, Suter MJF. Occurrence and fate of antibiotics as trace contaminants in wastewaters, sewage sludges, and surface waters. Chimia 57 (2003) 485-491.

3. Kolpin D, Furlong ET, Meyer MT, Thurman EM, Zaugg SD, Barber LB, Buxton HT. Pharmaceuticals, hormones, and other organic waste water contaminants in U.S. streams, 1999-2000: A national reconnaissance. Environmental Science \& Technology 36 (2002) 1202-1211.

4. Golet EM, Strehler A, Alder AC, Giger W. Determination of fluoroquinolone antibacterial agents in sewage sludge and sludgetreated soil using accelerated solvent extraction followed by solidphase extraction. Analytical Chemistry 74 (2002) 5455-5462.

5. Palmer AC, Angelino E, Kishony R. Chemical decay of an antibiotic inverts selection for resistance. Nature Chemical Biology 6 (2010) 105-107.

6. Karaseva N, Ermolaeva T, Mizaikoff B. Piezoelectric sensors using molecularly imprinted nanospheres for the detection of antibiotics. Sensors and Actuators B: Chemical 225 (2016) 199-208.

7. Jalili R, Khataee A, Rashidi M-R, Razmjou A. Detection of penicillin $G$ residues in milk based on dual-emission carbondots and molecularly imprinted polymers. Food Chemistry 314 (2020) 126172 .

8. Kwon D, Yoo H, Lee H, Jeon S. Colorimetric detection of penicillin $G$ in milk using antibody-functionalized dendritic platinum nanoparticles. Sensors and Actuators B 255 (2018) 552-556.

9. Weber P, Riegger BR, Niedergall K, Tovar GEM, Bach M, Gauglitz G, Nano-MIP based sensor for penicillin G: Sensitive layer and analyticalvalidation. Sensors and Actuators B 267 (2018) 26-33.

10. Pupin RR, Foguel MV, Gonçalves LM, Sotomayor MPT, Magnetic molecularly imprinted polymers obtained by photopolymerization for selective recognition of penicillin G. Journal applied polymer science 137 (2020) 48496.

11. Söylemez MA, Güven O. Preparation and detailed structural characterization of Penicillin G imprinted polymers by PALS and XPS. Radiation Physics and Chemistry 159 (2019) 174-180.

12. Kansy J. Microcomputer program for analysis of positron annihilation lifetime spectra. Nuclear Instruments and Methods in Physics Research Section A 374 (1996) 235-244.

13. Li H, Hu J, Wang C, Wang X. Removal of amoxicillin in aqueous solution by a novel chicken feather carbon: kinetic and equilibrium studies. Water Air Soil Pollution 228 (2017) 201.

14. Tsai H-J, Su Y-Y, Tseng C-C, Hsu W-K. Selective modification of aligned carbon nanotubes by N2 plasma and their diode behavior. RSC Advance 8 (2018) 10680-10685.

15. Söylemez MA, Güven O. Radiation induced in-situ synthesis of 
membranes for removal of 2,4-dichlorophenoxy acetic acid from real water samples. Radiation Physics and Chemistry 171 (2020) 108708.

16. Mkhize DS, Nyoni H, Quinn LP, Mamba BB, Msagati TA Molecularly imprinted membranes (MIMs) for selective removal of polychlorinated biphenyls (PCBs) in environmental waters: fabrication and characterization. Environmental Science and Pollution Research 24 (2017) 11694-11707.

17. Yoshimi Y, Namayama S, Piletsky SA. Changes in the porosity and permeability of a molecularly imprinted membrane induced by the adsorption of a trace quantity of template. The Open Analytical Chemistry Journal 7 (2013) 22-29.

18. Li FY, Li Y, Chung TS, Chen H, Jean YC, Kawi S. Development and positron annihilation spectroscopy (PAS) characterization of polyamide imide (PAI)-polyethersulfone (PES) based defectfree dual-layer hollow fiber membranes with an ultrathin denseselective layer for gas separation. Journal of Membrane Science 378 (2011) 541 .

19. Awad S, Chen HM, Grady BP, Paul A, Ford WT, Lee LJ, Jean YC Positron annihilation spectroscopy of polystyrene filled with carbon nanomaterials. Macromolecules 45 (2012) 933.

20. Positron Annihilation in Semiconductors: Defect Studies, Reinhard Krause-Rehberg, Hartmut S. Leipner, page: 1-2, C Springer-Verlag Berlin Heidelberg, 1999, Printed in Germany.

21. Wu X-B, Fan K-Q, Wang Q-H, Yang K-Q. C-terminus mutations of Acremonium chrysogenum deacetoxy/deacetylcephalosporin $\mathrm{C}$ synthase with improved activity toward penicillin analogs. FEMS Microbiology Letters 246 (2005) 103-110.

22. Umpleby RJ, Baxter SC, Chen Y, Shah RN, Shimizu KD Characterization of molecularly imprinted polymers with the Langmuir-Freundlich isotherm. Analytical Chemistry 73 (2001) 4584-4591.

23. Rampey AM, Umpleby II RJ, Rushton GT, Iseman JC, Shah RN, Shimizu KD. Characterization of the imprint effect and the influence of imprinting conditions on affinity, capacity, and heterogeneity in molecularly imprinted polymers using the Freundlich isotherm-affinity distributions analysis. Analytical Chemistry 76 (2004) 1123-1133.

24. Söylemez MA, Barsbay M, Güven O. Preparation of well-defined erythromycin imprinted non-woven fabrics via radiation-induced RAFT-mediated grafting. Radiation Physics and Chemistry 142 (2018) 77-81.

25. Sajini T, Gigimol MG, Mathew B. Kinetic and thermodynamic studies of molecularly imprinted polymers for the selective adsorption and specific enantiomeric recognition of D-mandelic acid. Journal of Polymer Research 26 (2019) 88.

26. Lakshmi D, Akbulut M, Ivanova-Mitseva PK, Whitcombe MJ, Piletska EV, Karim K, Güven O, Piletsky SA. Computational design and preparation of MIPs for atrazine recognition on a conjugated polymercoated microtitre plate. Industrial \& Engineering Chemistry Research 52 (2013) 13910-13916.

27. Ansell RJ, Mosbach K. Magnetic molecularly imprinted polymer beads for drug radioligand binding assay. Analyst 123 (1998) 16111616.

\section{APPENDIX}

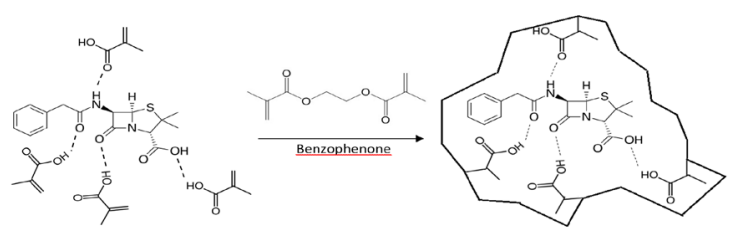

Figure S1. Synthesis of penicillin G imprinted polymers by employing methacrylic acid and ethylene glycol dimethacrylate as functional monomer and crosslinking agent, respectively.

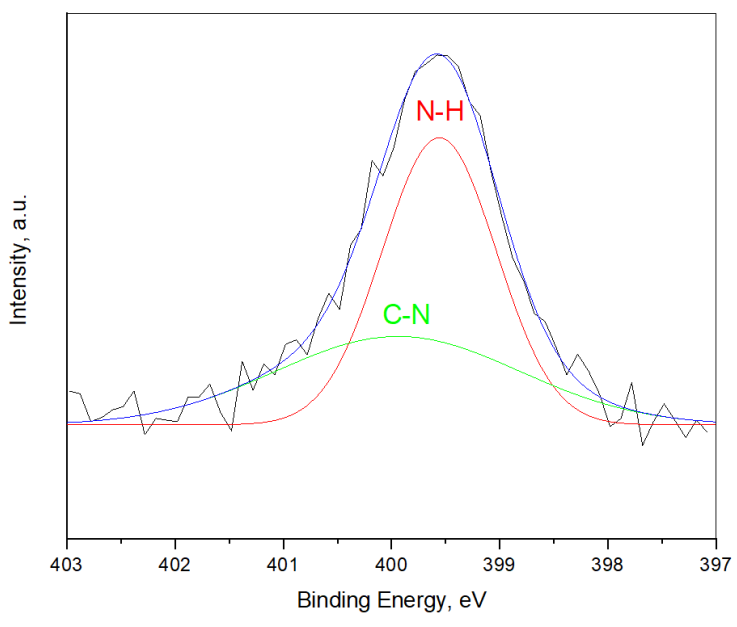

Figure S2. N1s spectrum of penicillin G imprinted membrane (MIM)

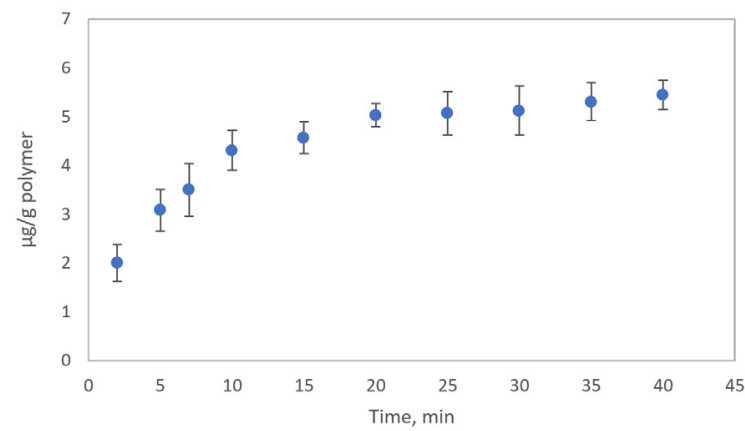

Figure S3. The binding capacity of the pencillin G imprinted membranes vs time. 
Table S1. Adsorption isotherm models and related parameters.

\begin{tabular}{|c|c|c|}
\hline Isotherm Model & Equation & Parameter \\
\hline Langmuir & $q_{e}=\frac{N K c_{e}}{1+K c_{e}}$ & $\begin{array}{l}\mathrm{q}_{\mathrm{e}} \text {, bound concentration of analyte on } \\
\text { imprinted membrane }\end{array}$ \\
\hline Scatchard Equation & $\frac{q_{e}}{c_{e}}=\mathrm{KN}-\mathrm{K} q_{e}$ & $\begin{array}{l}\mathrm{C}_{\mathrm{e}} \text {, free concentration of analyte in solution } \\
\mathrm{N} \text {, number of binding sites } \\
\mathrm{K} \text {, binding constant }\end{array}$ \\
\hline Freundich & $q_{e}=k_{f} c_{e}^{\mathrm{n}}$ & $\begin{array}{l}\mathrm{k}_{\mathrm{f}} \text {, adsorption constant } \\
\mathrm{n} \text {, heterogeneity index }\end{array}$ \\
\hline
\end{tabular}

Lagergren's first-order rate equation and pseudo second-order rate equation [1] were employed to evaluate the kinetics of the binding by using the equations which summarized in Table S2. The binding capacity of the membranes changes with time as apparent in Fig. S3.

Table S2. Kinetic models and related parameters.

\begin{tabular}{lll}
\hline Kinetic Model & Equation & Parameter \\
\hline $\begin{array}{l}\text { Pseudo-First Order Kinetic } \\
\text { Model }\end{array}$ & $\ln \left(\mathrm{q}_{\mathrm{e}}-\mathrm{q}_{\mathrm{t}}\right)=\ln \left(\mathrm{q}_{\mathrm{e}}\right)-\mathrm{k}_{1} \mathrm{t}$ & $\begin{array}{l}\mathrm{q}_{\mathrm{e}} \text {, amount of the analyte adsorbed at equilibrium } \\
\mathrm{q}_{\mathrm{t}} \text {, amount of the analyte adsorbed at time } \mathrm{t} \\
\mathrm{t} \text {, time } \\
\mathrm{k}_{1} \text {, pseudo-first order rate constant } \\
\mathrm{k}_{2} \text {, pseudo-second order rate constant }\end{array}$ \\
$\begin{array}{l}\text { Pseudo-Second Order Kinetic } \\
\text { Model }\end{array}$ & $\frac{\mathrm{t}}{\mathrm{q}_{\mathrm{t}}}=\left(\frac{1}{\mathrm{k}_{2} \mathrm{q}_{\mathrm{e}}^{2}}\right)+\left(\frac{1}{\mathrm{q}_{\mathrm{e}}}\right) \mathrm{t}$ & \\
\hline
\end{tabular}

Periasamy K, Namasvayam C. Process development for removal and recovery of cadmium from waste water by a low cost adsorbent: adsorption rate and equilibrium studies. Ind. Eng. Chem. Res. 1994, 33, 317-320.

Table S3. Equations and related parameters for dissociation constant $(\mathrm{Kd})$, selectivity $(\mathrm{k})$ and relative selectivity coefficient (k').

\begin{tabular}{|c|c|c|}
\hline & Equation & Parameter \\
\hline Dissociation Constant $\left(K_{d}\right)$ & $\mathrm{K}_{d}=\frac{\left(\mathrm{C}_{0}-\mathrm{C}_{f}\right)}{\mathrm{C}_{f}} x \frac{V}{m}$ & \\
\hline Selectivity Coefficient (k) & $\mathrm{k}=\frac{\mathrm{K}_{d \text { Penicillin } G}}{\mathrm{~K}_{\text {dAntibiotic }}}$ & $\begin{array}{l}\mathrm{C}_{0} \text { Initial concentration of dye } \\
\mathrm{C}_{\mathrm{f}} \text { Final concentration of dye } \\
\mathrm{V} \text { volume of the solution } \\
\mathrm{M} \text { mass of the dry polymer } \\
\mathrm{k}_{\mathrm{MIM}} \text { Selectivity coefficient of imprinted membrane } \\
\mathrm{k}_{\mathrm{NIM}} \text { Selectivity coefficient of non-imprinted membrane }\end{array}$ \\
\hline $\begin{array}{l}\text { Relative Selectivity Coeffici- } \\
\text { ent }\left(k^{\prime}\right)\end{array}$ & $\mathrm{k}^{\prime}=\frac{\mathrm{k}_{\mathrm{MIM}}}{\mathrm{k}_{\mathrm{NIM}}}$ & \\
\hline
\end{tabular}

Table S4. Dissociation constant $\left(\mathrm{K}_{\mathrm{d}}\right)$ and selectivity selectivity $(\mathrm{k})$ and relative selectivity coefficient (k').

\begin{tabular}{cccccc}
\hline & $\mathbf{K}_{\mathrm{d}, \mathrm{MIM}}$ & $\mathbf{K}_{\mathrm{d}, \mathrm{NIM}}$ & $\mathbf{k}_{\mathrm{MIM}}$ & $\mathbf{k}_{\mathrm{NIM}}$ & $\mathbf{k}^{\prime}$ \\
\hline Penicillin G & 4.75 & 2.04 & - & - & - \\
Penicillin V & 2.04 & 1.20 & 2.33 & 1.69 & 1.38 \\
Amoxycillin & 1.36 & 0.85 & 3.48 & 2.39 & 1.46 \\
\hline
\end{tabular}

